American Journal of Engineering and Applied Sciences 4 (4): 607-616, 2011

ISSN 1941-7020

(C) 2014 L. Chen et al., This open access article is distributed under a Creative Commons Attribution

(CC-BY) 3.0 license

\title{
Distributed Amplify and Forward Cooperation While Maintaining Transmission Freedom
}

\author{
${ }^{1}$ L. Chen, ${ }^{2}$ R. Carrasco and ${ }^{3}$ I. Wassell \\ ${ }^{1}$ School of Information Science and Technology, \\ Sun Yat-sen University, Guangzhou, 510006, China \\ ${ }^{2}$ School of Electrical, Electronic and Computer Engineering, Newcastle University, \\ Newcastle-upon-Tyne, NE1 7RU, United Kingdom \\ ${ }^{3}$ Digital Technology Group, Computer Laboratory, University of Cambridge, \\ CB3 OFD, Cambridge, United Kingdom
}

\begin{abstract}
Problem statement: This study proposes a novel Distributed Amplify and-Forward (DAF) cooperative scheme, achieving higher diversity order and yet maintaining the same transmission freedom as the conventional Amplify-and-Forward (AF) scheme. In the DAF scheme, a user's transmitted symbols are partitioned into several sequences in order to be relayed by different users. Approach: In the cooperative network, each user still uses half of their transmission for relaying others' signals. But instead of relaying one user's entire transmitted sequence, it helps different users for the relaying. Theoretical analysis of the DAF scheme is carried out in order to justify its advantages over the existing schemes. The outage behavior and Diversity-Multiplexing Tradeoff (DMT) analyses of the DAF scheme are presented. Results: Through outage behavior analysis, it is shown the DAF scheme achieves substantial diversity gains over the AF scheme. Furthermore, the DMT analysis justices both the scheme's achievable diversity gains and its ability to maintain the same multiplexing gain as the AF scheme. The theoretical analyses are then extended to a general cooperative network consisting of $\mathrm{N}(\mathrm{N} \geq 2)$ relays, showing the diversity order can be increased with respect to the number of relays but not at the expense of each user's multiplexing gain. Conclusion: Finally, a practical coded cooperative system that integrates the DAF scheme with the concatenated Reed-Solomon Convolution Codes (RSCC) is proposed, validating the achievable performance gain offered by the DAF scheme.
\end{abstract}

Key words:Multiple Input Multiple Output (MIMO), Amplify-and-Forward, distributed cooperation, diversity-multiplexing tradeoff, outage probability, concatenated Reed-Solomon convolution code, Decode-and-Forward (DF)

\section{INTRODUCTION}

Spatial diversity is a crucial technique to improve communication quality. The Multiple Input Multiple Output (MIMO) system (Alamouti, 1998) and the cooperative system (Laneman et al., 2004) are introduced due to their nature of creating spatial diversity. Among them, the cooperative system can be applied more widely since it does not impose any size constraint and extra cost to the mobile unit. In the cooperative communication network, each user equipped with a single antenna not only transmits their own information, but also relays other's information, creating a virtual multiple transmit antennas array. So far, there are three types of cooperative schemes: Amplify-and-Forward (AF) (Laneman and Wornell,
2000), Decode-and-Forward (DF) (Laneman and Wornell, 2000; Laneman et al., 2004) and Coded Cooperation (CC) (Hunter and Nosratinia, 2006; Stefanov and Erkip, 2004). The authors have carried out a comparative investigation of the existing schemes in (Chen et al., 2008).

For a cooperative network, each user not only transmits symbols carrying its own information bits, but also transmits symbols carrying others' information bits. Hence, in a cooperative network, transmission freedom is denned as the ratio of the transmission carrying one's own information bits to its total amount of transmission. User cooperation enables each user to achieve diversity gains, but it is also at the expense of their transmission freedom. In a practical communication system, this freedom loss is translated

Corresponding Author: L. Chen, School of Information Science and Technology, Sun Yat-sen University, Guangzhou, China, 510006 
into spectral efficiency loss. In this study, spectral efficiency is defined as the number of one's information bits carried by each of its transmitted symbol. To compensate, Chatzigeorgiou et al. (2008) proposed a high-order modulation scheme to be employed in the cooperative system. Later, the authors proposed Trellis Coded Modulation (TCM) scheme (Ungerboeck, 1982), achieving better coding gains for high spectral efficiency systems (Chen et al., 2009). Laneman and Wornell (2003) showed that better diversity gains can be achieved if cooperation is performed in a distributed manner, meaning more relays are involved for signal retransmission. Laneman and Wornell (2003), two types of distributed cooperation schemes were proposed: Repetition-based cooperation and spacetime-coded cooperation. For the repetition-based cooperation, extra spatial diversity is created at the cost of extra transmission freedom loss. As a result, the achievable diversity gain cannot be increased according to the number of users. For space-time-coded cooperation, the transmission freedom loss does not apply and hence diversity gains could be further achieved accordingly. Space-time-coded cooperation is operated in DF mode requiring decoding and reencoding at the relays. The diversity gain is achieved with substantial system complexity increase. Therefore, providing a distributed cooperative scheme without sacrificing transmission freedom or system complexity inspires the design of the DAF scheme.

This study introduces the DAF cooperative scheme, in which each user uses half of their transmission to relay more than one other user. To achieve this, the transmitted sequence of a user is partitioned into several parts, each of which is relayed by a different user in AF mode. Therefore, diverse transmission paths are created for the relayed symbols while each user still maintains their transmission freedom, half of that in noncooperation scenario. Theoretical analysis of the DAF scheme is presented in order to verify its performance advantage. Our analysis is drawn from a network with two relaying users and then extended to a larger network with $\mathrm{N}(\mathrm{N} \geq 2)$ users. The outage behavior analysis of the DAF scheme shows that substantial diversity gains could be achieved over the conventional AF scheme. The achievable diversity gains can be increased with respect to the number of relays. The DMT analysis was first introduced by Zheng and Tse (2003), analyzing the balance between the performance gain and the transmission freedom loss in MIMO systems. It was then applied to cooperative systems in Laneman et al. (2004); Laneman and Wornell (2003) and Azarian et al. (2005). Our DMT analysis shows that the DAF scheme has the same maximal multiplexing gain as the $\mathrm{AF}$ scheme, but achieves further diversity gains. The diversity gain is increased according to the number of relaying users. Prior to the writing of this study, the authors' earlier work (14) of integrating the DAF scheme with channel coding showed significant coding gains can be achieved over the AF scheme. In this study, the DAF scheme is integrated with a widely used concatenated errorcorrection code, the concatenated Reed-Solomon Convolution Code (RSCC). The simulation results show significant performance gain can be achieved for a practical coded system.

The study is organized as follows: In the bigninig presented the preliminaries of the study, then presented the DAF system model, after that presented the outage behavior analysis; the at the end presented the DMT analysis. Both the outage behavior analysis and DMT analysis are extended in a larger cooperative network in a RSCC coded DAF system with performance evaluation. Finally, concludes the study.

Preliminary: This study presents the preliminaries of the study. It includes dentitions of commonly used parameters and an introduction to the conventional AF scheme which will be used to compare with the proposed DAF scheme.

Parameterizations: The analyzed cooperative network is assumed to operate in half-duplex mode, requiring orthogonal time division channel allocation for the receiving and transmitting of each user. The channel quality is measured by the transmitted Signal-to-Noise Ratio (SNR) which can be defined as Eq. 1:

$\rho=\frac{\varepsilon}{\sigma^{2}}$

where, $\varepsilon$ denotes the average transmitted symbol energy and $\sigma^{2}$ denotes the variance of noise at the receiver. For simplicity of the analysis, it is assumed that the network has symmetric channels meaning all of them have similar SNR values. The channel between transmitting user and receiving user $\mathrm{b}$ is assumed to be Quasi-static Rayleigh fading with fading coefficient. All channels within the cooperative network are assumed to be statistically independent. $\alpha_{a b}$ is a Gaussian random variable with zero mean and unit variance. The exponential order of $1 /\left|\alpha_{\mathrm{ab}}\right|^{2}$ is defined as Eq. 2:

$$
\delta a b=\lim _{\rho \rightarrow \infty} \frac{R(\rho)}{\log \rho}
$$

where, the base of the logarithm is 2 . 
If the cooperative system operates with a transmission rate of $\mathrm{R}(\rho)$ bits sec $\mathrm{Hz}^{-1}$, which is a function of the SNR, the multiplexing gain of the system can be defined as Eq. 3:

$$
r=\lim _{\rho \rightarrow \infty} \frac{R(\rho)}{\log \rho}
$$

where, $\mathrm{r}$ is a normalised value representing the ratio of effective transmission. At a SNR of $\rho$, if the system can achieve a Maximum-Likelihood (ML) error probability of $\mathrm{P}_{\mathrm{e}}(\rho)$, its diversity gain is defined as Eq. 4 (Zheng and Tse, 2003):

$$
d=\lim _{\rho \rightarrow \infty} \frac{\log (\operatorname{Pe}(\rho))}{\log \rho}
$$

The derived result of the relationship between $d$ and $r$ is called the Diversity-Multiplexing Tradeoff (DMT), denoted as $\mathrm{d}(\mathrm{r})$. We could further claim protocol A is superior to protocol B if for any multiplexing gain $r, d_{A}(r) \geq d_{B}(r) . \Re^{N}$ and $C^{N}$ denote the set of real and complex $\mathrm{N}$-tulles. $\mathrm{N}+$ denotes the set of nonnegative $\mathrm{N}$-tulles. $\mathrm{O}$ is used to denote the set of outage events in the cooperative system, $\mathrm{O} \subseteq \mathfrak{R}^{\mathrm{N}}$ and $\mathrm{O}^{+}=\mathrm{O} \cap \Re^{\mathrm{N}+}$. According to (Zheng and Tse, 2003; Azarian et al., 2005), the DMT (d(r)) of a cooperative system with $\mathrm{N}$ users is upper bounded by Eq. 5 (Azarian et al., 2005):

$$
\mathrm{d}(\mathrm{r}) \leq \mathrm{d}_{0} \text { and } \mathrm{d}_{0}=\quad \underset{(\delta 1, \ldots, \delta \mathrm{W}) \in \mathrm{O}^{+}}{\sum_{\mathrm{j}=1}^{\mathrm{W}} \delta \mathrm{j}}
$$

where, $\mathrm{j}$ is the index of all the $\mathrm{W}$ channels of the cooperative network. The analyses result of this study is given when $d(r)$ saturates the bound. Note that in the rest of the study, $\mathrm{N}$ means the number of the relaying users excluding the transmit user. $\mathrm{P}_{\mathrm{DAF}(\mathrm{N})}$ out and $\mathrm{d}$ $\operatorname{DAF}_{(\mathrm{N})}(\mathrm{r})$ denote the outage probability and DMT of the DAF scheme with $\mathrm{N}$ relaying users. Furthermore, $\mathrm{I}_{\mathrm{N}}$ denotes the $\mathrm{N} \times \mathrm{N}$ identity matrix, $\mathrm{d}_{\mathrm{et}}(\mathrm{x})$ denotes the determinant of the matrix $\mathrm{x}, \sum_{\mathrm{x}}$ denotes the auto covariance matrix of vector $\mathrm{x}, \mathrm{x}^{\mathrm{H}}$ denotes the Hermitian conjugates of matrix $\mathrm{x}$ and $(\mathrm{x})^{+}$means $\max \{\mathrm{x}, 0\}$.

Amplify-and-forward: The AF scheme consists of three users: Source (S) and its signal Destination (D), Relay (R) helps $\mathrm{S}$ for the transmission. A classical cooperative process contains two Time Slots (TS) with equal duration. The first TS is for initial transmission when $\mathrm{S}$ transmits its information to $\mathrm{D}$ and $\mathrm{R}$. The second TS is for relaying transmission when $R$ amplifies its received signal from $\mathrm{S}$ and transmits it to D. D would combine the received signals using ML detection (Laneman and Wornell, 2000). If $\alpha_{\mathrm{SD}}, \alpha_{\mathrm{SR}}$ and $\alpha_{\mathrm{RD}}$ denote the fading coefficients of the channels between S-D, S-R and R-D respectively and R denotes the transmission rate of the cooperative system, the outage behavior of the AF scheme can be modeled as Eq. 6 and 7 (Laneman et al., 2004):

$$
\mathrm{P}_{\mathrm{AF}}^{\text {out }}=\operatorname{Pr}\left[1+\left|\alpha_{\mathrm{SD}}\right|^{2} \rho+\mathrm{f}\left(\left|\alpha_{\mathrm{SR}}\right|^{2} \rho,\left|\alpha_{\mathrm{RD}}\right|^{2} \rho\right)<2^{2 \mathrm{R}}\right]
$$

Where:

$$
f(\mu, v)=\frac{\mu v}{\mu+v+1}
$$

$\mu$ And $v$ are random variables. The DMT characteristics of the AF scheme can be described by Eq. 8 (Laneman et al., 2004):

$$
\mathrm{dAF}(\mathrm{r}) \leq 2(1-2 \mathrm{r})^{+}
$$

It can be seen that diversity order of 2 can be obtained from the AF scheme.

System model: This model presents the system model for the DAF scheme, detailing this novel transmission protocol. In general, if a DAF cooperative network has $\mathrm{N}$ relaying users, the transmitted signal of $\mathrm{S}$ will be equally partitioned into $\mathrm{N}$ sections, each of which will be relayed by a different user. It is not difficult to realize that when $\mathrm{N}=1$, it becomes the conventional AF scheme. For simplicity, the description of the DAF system model is given with $\mathrm{N}=2$ and it could be easily extended into a larger cooperative network. A complete cooperative process of the DAF scheme also consists of two TSs, which is shown in Fig. 1. In the first TS, transmits its signal to $\mathrm{D}$ as well as to two different relays $\left(R_{1}\right.$ and $\left.R_{2}\right)$. It is assumed that $R_{1}$ and $R_{2}$ are perfectly synchronized with $S$ and $R_{1}$ received the first half of $S$ ' ${ }_{S}$ signal while $R_{2}$ received the second half.

In the following equations, signals $(x, y, w, v)$ have double subscripts $(a, b)$ where denotes the TS that the signal belongs to and $\mathrm{b}$ denotes the symbol index. $\mathrm{x}$ denotes the transmitted signal and $y$ denotes the received signal. $\mathrm{v}$, wand ware the Additive White Gaussian Noise (AWGN) at D, $\mathrm{R}_{1}$ and $\mathrm{R}_{2}$ respectively. They are modeled as mutually independent, zero-mean complex random sequence with variances $\sigma^{2}{ }_{v}, \sigma^{2}{ }_{w 1}$ and $\sigma^{2}{ }^{2}$ respectively. The S-D transmission can be described Eq. 9 as:

$$
\mathrm{y}_{1, \mathrm{k}}=\alpha_{\mathrm{SD}} \mathrm{x}_{1, \mathrm{k}}+\mathrm{v}_{1, \mathrm{k}}, \mathrm{k}=1,2, \ldots, 1 / 2
$$

where, 1 denotes the length of the signal transmitted during the two $\mathrm{TS}_{\mathrm{s}}$ and $\mathrm{l} \mid 2 \mathrm{~N}$. 

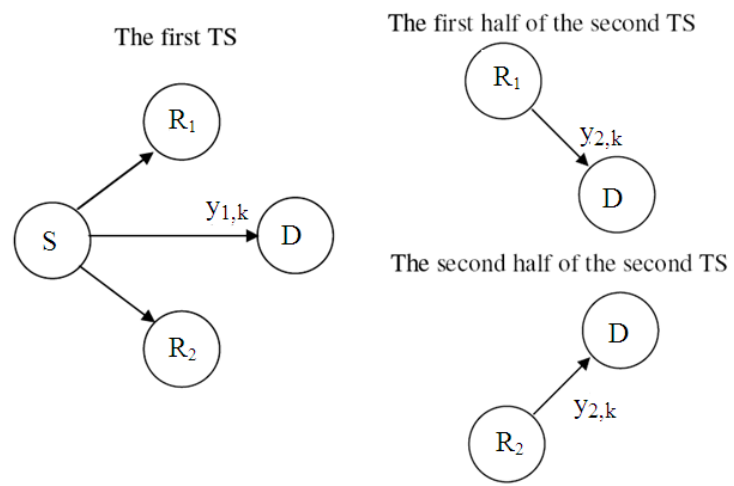

Fig. 1: Cooperation process for the DAF scheme

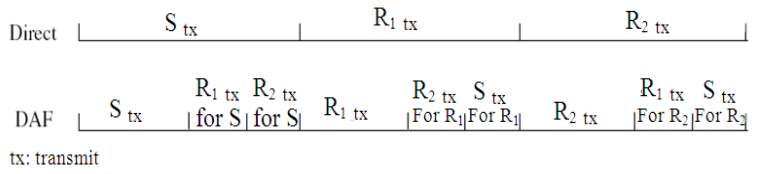

Fig. 2: Time division channel allocation for the DAF scheme with $\mathrm{N}=2$

The second TS are also partitioned into two equal halves for relaying transmission. In the first half of the second TS, $R_{1}$ amplifies it's received signal with gain $\beta_{1}$ and re-transmits to D as Eq. 10 and 11:

$$
\begin{aligned}
& \mathrm{y}_{2, \mathrm{k}}=\alpha \mathrm{R}_{1} \mathrm{D}^{\beta_{1}}\left(\alpha_{\mathrm{SR} 1} \mathrm{x}_{1}, \mathrm{k}_{\mathrm{k}-1 / 2}+\mathrm{w}_{1_{\mathrm{k}-1 / 2}}\right)+\mathrm{v}_{2, \mathrm{k}}, \\
& \mathrm{k}=1 / 2+1,1 / 2+2, \ldots, 31 / 4
\end{aligned}
$$

Where:

$$
\beta_{1} \leq \sqrt{\frac{\varepsilon}{\left|\alpha_{\mathrm{SR} 1}\right|^{2} \varepsilon+\sigma^{2}{ }_{\mathrm{w} 1}}}
$$

Similarly, in the second half of the second TS, $\mathrm{R}_{2}$ re-transmit $\mathrm{S}_{\mathrm{s}}$ signal to $\mathrm{D}$ as Eq. 12 and 13:

$$
\begin{aligned}
& \mathrm{y}_{2, \mathrm{k}}=\alpha \mathrm{R}_{2} \mathrm{D}_{2}\left(\alpha \mathrm{SR}_{2} \mathrm{x}_{1, \mathrm{k}-1 / 2}+\mathrm{w}_{1, \mathrm{k}-1 / 2}\right)+\mathrm{v}_{2, \mathrm{k}}, \\
& \mathrm{k}=31 / 4+1,31 / 4+2, \ldots, 1,
\end{aligned}
$$

Where:

$$
\beta_{2} \leq \sqrt{\frac{\varepsilon}{\left|\alpha_{\mathrm{SR} 2}\right|^{2} \varepsilon+\sigma^{2}{ }^{2} 2}}
$$

After the two $\mathrm{TS}_{\mathrm{s}}, \mathrm{D}$ combines $\mathrm{y}_{1, \mathrm{k}}(\mathrm{k}=1,2, \ldots$, 1/4) with $\mathrm{y}_{2, \mathrm{k}}$ of $(10)$ and $\mathrm{y}_{1, \mathrm{k}}(\mathrm{k}=1 / 4+1,1 / 4+2, \ldots, 1 / 2)$ with $\mathrm{y}_{2, \mathrm{k}}$ of (12) for further signal processing in order to retrieve the transmitted information. Figure 2 shows the time division channel allocation structure of the DAF scheme. It can be seen that half of a user's total transmission is used for their own transmission, while the other half is partitioned into smaller divisions in order to help different users. It maintains the same transmission freedom as the conventional $\mathrm{AF}$ scheme (Laneman et al., 2004).

Outage behavior: This study presents the outage behavior analysis for the DAF scheme with two relaying users. Its extension to larger networks will be mentioned in Section VI. The conclusion is drawn by first formalizing the transmission signal model, then determining the scheme's mutual information and Nelly modeling its outage behavior. The following theorem models the scheme's outage behavior.

Theorem 1: For a DAF cooperative scheme with two relaying users, if its transmission rate is $\mathrm{R}$ bits sec $\mathrm{Hz}^{-1}$, its outage behavior can be determined by Eq. 14:

$$
\mathrm{P}_{\mathrm{DAF}(2)}^{\text {Out }}=\operatorname{Pr}\left[\begin{array}{l}
\left.\prod_{i=1}^{2} 1+|\alpha \mathrm{SD}|^{2} \rho+\mathrm{f}\left(\left|\alpha \mathrm{SR}_{\mathrm{t}}\right|^{2} \rho,\left|\alpha \mathrm{R}_{\mathrm{t}} \mathrm{D}\right|^{2} \rho\right)\right] \\
<2^{4 R}
\end{array}\right]
$$

Proof: To prove Theorem 1, it is necessary to formalize the system model of the DAF scheme into matrix form. Equations $9-13$ can be alternatively expressed Eq. 15 as:

$$
\begin{aligned}
& \bar{y}=\left[\begin{array}{cc}
\alpha_{\mathrm{SD}} \mathrm{I}_{1 / 4} & 0 \\
0 & \alpha_{\mathrm{SD}} \mathrm{I}_{1 / 4} \\
\alpha \mathrm{R}_{1} \mathrm{D} \beta_{1} \alpha \mathrm{SR}_{1} \mathrm{Il} / 4 & 0 \\
0 & \alpha \mathrm{R}_{2} \mathrm{D} \beta_{2} \alpha \mathrm{SR}_{2} \mathrm{Il} / 4
\end{array}\right] \overline{\mathrm{x}} \\
& +\left[\begin{array}{cc}
0 & 0 \\
0 & 0 \\
\alpha \mathrm{R}_{1} \mathrm{D} \beta_{1} \mathrm{Il} / 4 & 0 \\
0 & \alpha \mathrm{R}_{2} \mathrm{D} \beta_{2} \mathrm{Il} / 4
\end{array}\right] \overline{\omega_{2}}+\bar{v}
\end{aligned}
$$

where, $\bar{x} \in C^{1 / 2 \times 1}$ denotes vector of transmitted signals, $\bar{y} \in C^{1 \times 1}$ denotes the vector of received signals at $D$, $\bar{v} \in C^{1 \times 1}$ denotes the vector of noise samples at $\mathrm{D}$ and $\overline{\omega l}$ and $\overline{\omega 2} \in \mathrm{C}^{1 / 4 \times 1}$ denote the vector of noise samples at $R_{1}$ and $R_{2}$. Let $x_{i}$ and $y_{i}$ denote the entices of $\bar{x}$ and $\bar{y}$, the relationship between $x_{i}$ and $y_{i}$ can be categorised into the following two sets. For $\mathrm{i}=1,2 \ldots$. $1 / 4$ as Eq. 16-21: 
$\hat{y}_{\mathrm{i}}^{1}=\left[\begin{array}{c}\mathrm{y}_{\mathrm{i}} \\ \mathrm{y}_{\mathrm{i}}+1 / 2\end{array}\right]=\overline{\mathrm{G}}_{\mathrm{i}}^{1} \mathrm{x}_{\mathrm{i}}+\hat{\mathrm{n}}_{\mathrm{i}}^{1}$,

Where:

$\mathrm{G}_{\mathrm{i}}^{1}=\left[\begin{array}{c}\alpha_{\mathrm{SD}} \\ \alpha \mathrm{R}_{1} \mathrm{D} \beta_{1} \alpha_{\mathrm{SR} 1}\end{array}\right]$,

$\sum_{\hat{\mathrm{n}}^{\mathrm{n}}}^{1}=\left[\begin{array}{cc}\sigma_{v}^{2} & \sigma_{v}^{2}+\mid \alpha \mathrm{R}_{1} \\ 0 & 0 \\ 0 & \left.|2| \beta_{1}\right|^{2} \sigma_{\mathrm{w} 1}^{2}\end{array}\right]$

Similarly for $\mathrm{i}=1 / 4+1,1 / 4+2 . .1 / 2$ :

$\hat{y}_{i}^{2}=\left[\begin{array}{c}y_{i} \\ y_{i}+1 / 2\end{array}\right]=\bar{G}_{i}^{2} x_{i}+\hat{n}_{i}^{2}$,

Where:

$$
\begin{aligned}
& \mathrm{G}_{i}^{2}=\left[\begin{array}{c}
\alpha_{\mathrm{SD}} \\
\alpha \mathrm{R}_{2} \mathrm{D} \beta_{2} \alpha_{\mathrm{SR} 2}
\end{array}\right] \\
& \sum_{\hat{\mathrm{n}^{\mathrm{n}}}}^{2}=\left[\begin{array}{ccc}
\sigma_{v}^{2} & \sigma_{v}^{2}+\mid \alpha \mathrm{R}_{1} & 0 \\
0 & \left.\right|^{2}\left|\beta_{2}\right|^{2} & \sigma_{\mathrm{w} 2}^{2}
\end{array}\right]
\end{aligned}
$$

Now, it is straight forward to see that the mutual information between $\overline{\mathrm{x}}$ and $\overline{\mathrm{y}}$ can be determined by Eq. 22:

$$
\mathrm{I}(\overline{\mathrm{x}}, \overline{\mathrm{y}})=\frac{1}{4}\left[\left(\mathrm{x}_{\mathrm{i}}, \hat{\mathrm{y}}_{\mathrm{i}}^{1}\right)+\mathrm{I}\left(\mathrm{x}_{\mathrm{i}}, \hat{\mathrm{y}}_{\mathrm{i}}^{2}\right)\right]
$$

The mutual information between $x_{i}$ and $\hat{y}_{i}^{1}$ determined by Eq. 23:

$$
\begin{aligned}
& I\left(x_{i}, \hat{y}_{i}^{1}\right)=\log \left(\operatorname{det}\left(I_{2}+x_{i} G_{i}^{1} G_{i}^{1 H} \sum_{\hat{n}_{i}^{1}}^{-1}\right)\right) \\
& \leq \log \left(\operatorname{det}\left(I_{2}+\varepsilon G_{i}^{1} G_{i}^{1 H} \sum_{\hat{n}_{i}^{1}}^{-1}\right)\right)
\end{aligned}
$$

$\mathrm{I}\left(\mathrm{x}_{\mathrm{i}}, \hat{\mathrm{y}}_{\mathrm{i}}^{1}\right)$ Can only be determined by saturating the bound in (23). By substituting Eq. 17 and 18 into 23, with a few algebraic manipulations, it can be derived that as Eq. 24:

$$
\mathrm{I}\left(\mathrm{x}_{\mathrm{i}}, \hat{\mathrm{y}}_{\mathrm{i}}^{1}\right)=\log \left(1+\frac{|\alpha \mathrm{SD}|^{2} \varepsilon}{\sigma_{v}^{2}}+\frac{\left|\alpha_{\mathrm{R} 1} \mathrm{D}\right|^{2}\left|\beta_{1}\right|^{2}\left|\alpha \mathrm{SR}_{1}\right|^{2} \varepsilon}{\sigma_{v}^{2}+\left|\alpha_{\mathrm{R} 1} \mathrm{D}\right|^{2}\left|\beta_{1}\right|^{2} \sigma_{\mathrm{w} 1}^{2}}\right)
$$

Through the same methodology, we can determine that as Eq. 25:

$$
\mathrm{I}\left(\mathrm{x}_{\mathrm{i}}, \hat{\mathrm{y}}_{\mathrm{i}}^{2}\right)=\log \left(1+\frac{|\alpha \mathrm{SD}|^{2} \varepsilon}{\sigma_{v}^{2}}+\frac{\left|\alpha_{\mathrm{R} 2} \mathrm{D}\right|^{2}\left|\beta_{2}\right|^{2}\left|\alpha \mathrm{SR}_{2}\right|^{2} \varepsilon}{\sigma_{v}^{2}+\left|\alpha_{\mathrm{R} 2} \mathrm{D}\right|^{2}\left|\beta_{2}\right|^{2} \sigma_{\mathrm{w} 2}^{2}}\right)
$$

Therefore, by substituting Eq. 24 and 25 into 22, we have Eq. 26:

$$
\mathrm{I}(\overline{\mathrm{x}}, \overline{\mathrm{y}})=\frac{1}{4} \sum_{\mathrm{t}=1}^{2} \log \left(1+\frac{\left|\alpha_{\mathrm{SD}}\right|^{2} \varepsilon}{\sigma_{v}^{2}}+\frac{\left|\alpha_{\mathrm{Rt}} \mathrm{D}\right|^{2}\left|\beta_{\mathrm{t}}\right|^{2}\left|\alpha \mathrm{SR}_{\mathrm{t}}\right|^{2} \varepsilon}{\sigma_{\mathrm{v}}^{2}+\left|\alpha_{\mathrm{Rt}} \mathrm{D}\right|^{2}\left|\beta_{2}\right|^{2} \sigma_{\mathrm{wt}}^{2}}\right)
$$

When $\beta_{1}$ and $\beta_{2}$ saturate the bounds in (11) and (13) and in a symmetric network implied as: $\frac{\varepsilon}{\sigma_{v}^{2}}=\frac{\varepsilon}{\sigma_{\mathrm{w} 1}^{2}}=\frac{\varepsilon}{\sigma_{\mathrm{w} 1}^{2}}=\rho$ Eq. 26 can be simplified as:

$$
\mathrm{I}(\overline{\mathrm{x}}, \overline{\mathrm{y}})=\frac{1}{4} \sum_{\mathrm{t}=1}^{2} \log \left(1+\left|\alpha_{\mathrm{SD}}\right|^{2} \rho+\mathrm{f}\left(\left|\alpha_{\mathrm{SRt}}\right|^{2} \rho,\left|\alpha \mathrm{R}_{\mathrm{t}} \mathrm{D}\right|^{2} \rho\right)\right)
$$

In a DAF system with transmission rate of $\mathrm{R}$, its outage behavior is determined by:

$\mathrm{P}_{\mathrm{DAF}(2)}^{\mathrm{Out}}=\operatorname{Pr}[\mathrm{I}(\overline{\mathrm{x}}, \overline{\mathrm{y}})<\mathrm{IR}]$

By substituting Eq. 27 into 28, it is not difficult to derive Eq. 14 and the proof is complete.

Figure 3 shows the Monte-Carlo simulation results comparing the outage behavior between the $\mathrm{AF}$ and DAF schemes. They are obtained by using Eq. 6-14 respectively. The cooperative systems are set with $\mathrm{R}=$ 0.5 and $\mathrm{R}=1$ bits $\mathrm{sec} \mathrm{Hz}^{-1}$. It can be seen that cooperation outperforms direct transmission. More importantly, the DAF scheme can achieve significant diversity gains over the $\mathrm{AF}$ scheme. For example, at outage probability of $10^{-5}$ the DAF scheme can achieve a $5 \mathrm{~dB}$ diversity gain over the $\mathrm{AF}$ scheme.

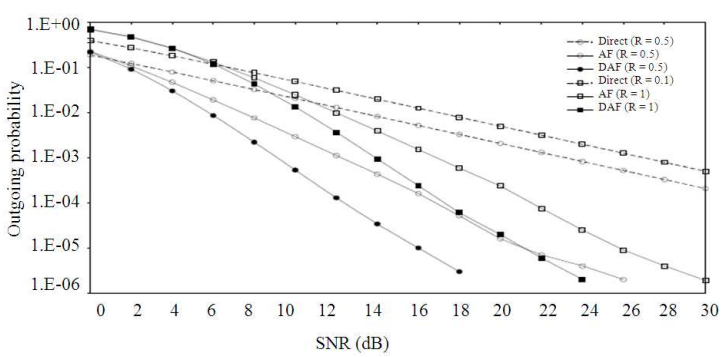

Fig. 3: Outage behavior analysis of the DAF scheme 
It is also important to emphasize that unlike most distributed cooperative schemes, this diversity gain is not achieved at the cost of each user's transmission freedom. Its ability to maintain the same transmission freedom as the AF scheme will be justice in the next step.

Diversity-multiplexing tradeoff: This segment presents the DMT analysis for the proposed scheme. Through the DMT analysis, we are able to determine the scheme's maximal multiplexing gain and diversity gain. The tradeoff between them can also be rejected from the analysis. First of all, the following theorem is proposed to describe the DMT of the scheme.

Theorem 2: For a DAF cooperative scheme with two relaying users, if its transmission rate is $\mathrm{R}$ bits $\mathrm{sec} \mathrm{Hz}^{-1}$, its diversity-multiplexing tradeoff is upper bounded by:

$$
\mathrm{d}_{\mathrm{DAF}(2)}(\mathrm{r}) \leq 3(1-2 \mathrm{r})^{+}
$$

Proof: Referring to Eq. 3 and 4, both d and r describe the system's asymptotic behavior when $\rho \rightarrow \infty$. To analyze $\mathrm{d}_{\mathrm{DAF}(2)}(\mathrm{r})$, we shall also analyze $\mathrm{I}(\overline{\mathrm{x}}, \overline{\mathrm{y}})$ 's asymptotic behavior since it is also a function of $\rho$. Based on Eq. 26, it can be derived that:

$$
\begin{aligned}
& \lim _{\rho \rightarrow \infty} \frac{\mathrm{I}(\overline{\mathrm{x}}, \overline{\mathrm{y}})}{\log \rho}=\lim _{\rho \rightarrow \infty} \frac{1}{4 \log \rho} \sum_{\mathrm{t}=1}^{2} \log \left(1+|\alpha \mathrm{SD}|^{2} \rho\right. \\
& +\frac{\left|\alpha \mathrm{R}_{\mathrm{t}} \mathrm{D}\right|^{2}|\beta \mathrm{t}|^{2}\left|\alpha \mathrm{SR}_{\mathrm{t}}\right|^{2} \rho}{1+\left|\alpha \mathrm{R}_{\mathrm{t}} \mathrm{D}\right|^{2}\left|\beta_{\mathrm{t}}\right|^{2} \sigma^{2}{ }_{\text {ot }} / \sigma_{v}^{2}} \\
& \quad \text { When } \rho \rightarrow \infty, \text { we could claim the following }
\end{aligned}
$$
approximations:

$$
\begin{aligned}
& \left|\beta_{\mathrm{t}}\right|^{2} \cong 1, \sigma_{\mathrm{wt}}^{2} / \sigma_{\mathrm{v}}^{2} \cong 1,|\alpha \mathrm{SD}|^{2} \cong \rho^{-\delta S \mathrm{SD}} \\
& \left|\alpha \beta_{\mathrm{t}}\right|^{2} \cong \rho^{-\delta S R t} \text { and }\left|\alpha \mathrm{R}_{\mathrm{t}} \mathrm{D}\right|^{2} \cong \rho^{-\delta \mathrm{RtD}}
\end{aligned}
$$

Therefore, Eq. 30 can be further simplified to Eq. 31:

$$
\lim _{\rho \rightarrow \infty} \frac{I(\bar{x}, \bar{y})}{\log \rho} \cong \frac{1 \sum_{t=1}^{2} \log \left(1+\rho^{1-\delta S D}+\rho^{1-(\delta S R} t^{+\delta R} t^{D)}\right)}{4 \log \rho}
$$

It can be further approximated as:

$$
\begin{aligned}
& \lim _{\rho \rightarrow \infty} \frac{\mathrm{I}(\overline{\mathrm{x}}, \overline{\mathrm{y}})}{\log \rho} \cong \\
& \frac{1}{4} \sum_{\mathrm{t}=1}^{2}\left(\max \left\{\frac{\log \rho^{1-\delta S \mathrm{D}}}{\log \rho}, \frac{\log \rho^{1-\delta S R_{\mathrm{t}}+\delta S R_{\mathrm{t}} \mathrm{D}}}{\log \rho}\right\}\right)+= \\
& \frac{1}{4} \sum_{\mathrm{t}=1}^{2}\left(\max \left\{1-\delta_{\mathrm{SD}}, 1-\left(\delta \mathrm{SR}_{\mathrm{t}}+\delta \mathrm{SR}_{\mathrm{t}} \mathrm{D}\right)\right\}\right)+
\end{aligned}
$$

Equation 32 links the mutual information with the exponential orders of each channel. The set of outage events is defined as the set of instantaneous channel realizations in which its mutual information falls below its transmission rate as:

$$
\left.\mathrm{O}=\left\{\left(\delta_{\mathrm{SD}}, \delta_{\mathrm{SRt}}, \delta \mathrm{R}_{\mathrm{t}} \mathrm{D}(\mathrm{t}=1,2)\right) \mid \mathrm{I}(\overline{\mathrm{x}}, \overline{\mathrm{y}})<\mathrm{RR}\right)\right\}
$$

Based on Eq. 32-34 knowing asymptotically $\mathrm{R}=\mathrm{r} \log$ $\rho$, we can further define:

$$
\begin{aligned}
& \mathrm{O}^{+}=\left\{\left(\delta_{\mathrm{SD}}, \delta_{\mathrm{SR} t}, \delta \mathrm{R}_{\mathrm{t}} \mathrm{D}(\mathrm{t}=1,2)\right) \in \mathfrak{R}^{3+} \mid\right. \\
& \left.\sum_{\mathrm{t}=1}^{2}\left(\max \left\{1-\delta_{\mathrm{SD}}, 1-\left(\delta_{\mathrm{SRt}}+\delta_{\mathrm{Rt}} \mathrm{D}\right)\right\}\right)^{+}<4 \mathrm{r}\right\}
\end{aligned}
$$

From Eq. 34, it can be calculated that $1-2 \mathrm{r}<\delta_{\mathrm{SD}}<1$ and

$$
2-4 \mathrm{r}<\sum_{\mathrm{t}=1}^{2}\left(\max \left\{1-\delta_{\mathrm{SD}}, 1-\left(\delta_{\mathrm{SRt}}+\delta_{\mathrm{Rt}} \mathrm{D}\right)\right\}\right)<2 .
$$

According to $\mathrm{d}(\mathrm{r})$ dentition given by Eq. 5, we can easily conclude its upper bound expression which is given by Eq. 29. The proof is complete. Furthermore, the scheme's capability of achieving such an upper bound is proven in Chen et al. (2011) to which the interest readers may refer.

Figure 4 shows the DMT analysis result of the DAF scheme. Its performance is compared with the conventional AF scheme with one and two relays respectively. Notice that the AF scheme with two relays is identical to the distributed cooperative scheme using repetition re-transmission proposed in (Laneman and Wornell, 2003). Observing this, we conclude the following comments: First, cooperation achieves further diversity gain compared to direct transmission, but loses multiplexing gain, i.e., loss of transmission freedom; second, the DAF scheme achieves the same maximal multiplexing gain as the AF scheme with one relay, but achieves higher diversity gain. It varies the achieved diversity gains shown in Fig. 3. Third, the DAF scheme achieves the same maximal diversity gain as the AF scheme with two relays, but achieves higher maximal multiplexing gain-maintaining higher transmission freedom compared to the existing distributed cooperative scheme.

Extension to multiple users network: This study extends the analysis proposed in the above two sections to a larger cooperative network with $\mathrm{N}(\mathrm{N} \geq 2)$ relaying users. Two theorems describing its outage behavior and diversity-multiplexing tradeoff will be presented. Since the same methodology to prove Theorem 1 and Theorem 2 is used, the proof given in this study will only state the important generalized equations. In order 
to substantiate the theorems, the corresponding simulation and analysis results will also be shown.

In a DAF cooperative network with $\mathrm{N}$ relaying users, $\mathrm{S}$ will partition its sequence into $\mathrm{N}$ equal parts of length $1 / 2 \mathrm{~N}$, each of which will be relayed by a different user. Its system model can be easily extended. The following theorem describes its outage behavior.

Theorem 3: For a DAF cooperative scheme with $\mathrm{N}$ (N $\geq 2$ ) relaying users, if its transmission rate is $\mathrm{R}$ bits sec $\mathrm{Hz}^{-1}$, its outage behavior can be determined by:

$\mathrm{P}_{\mathrm{DAF}(2)}^{\text {Out }}=\operatorname{Pr}\left[\begin{array}{l}\prod_{t=1}^{\mathrm{N}} 1\left(+|\alpha \mathrm{SD}|^{2} \rho+\mathrm{f}\left(\left|\alpha \mathrm{SR}_{\mathrm{t}}\right|^{2} \rho,\left|\alpha \mathrm{R}_{\mathrm{t}} \mathrm{D}\right|^{2} \rho\right)\right) \\ <2^{2 \mathrm{NR}}\end{array}\right](35)$

Proof: Similar to the proof of Theorem 1, by formalising its system model into matrix form, we can have the following $\mathrm{N}$ signal tuples $\left(\left(x_{i}, \hat{y}_{i}^{1}\right),\left(x_{i}, \hat{y}_{i}^{2}\right), \ldots .\left(x_{i}, \hat{y}_{i}^{N}\right)\right.$ with length $1 / 2 N$.

The mutual information between the transmitted signal $\bar{x}$ and received signal $\bar{y}$ can be determined by Eq. 36:

$\mathrm{I}(\overline{\mathrm{x}}, \overline{\mathrm{y}})=\frac{1}{2 \mathrm{~N}} \sum_{\mathrm{t}=1}^{\mathrm{N}} \mathrm{I}\left(\mathrm{x}_{\mathrm{i}}, \hat{\mathrm{y}}_{\mathrm{i}}^{\mathrm{t}}\right)$

Applying the derived results of Eq. 24 and 25, we have:

$\mathrm{I}(\overline{\mathrm{x}}, \overline{\mathrm{y}})=\frac{1}{2 \mathrm{~N}} \sum_{\mathrm{t}=1}^{\mathrm{N}} \log \left(1+\frac{\left|\alpha_{\mathrm{SD}}\right|^{2} \varepsilon}{\sigma_{\mathrm{v}}^{2}}+\frac{\left|\alpha_{\mathrm{Rt}} \mathrm{D}\right|^{2}\left|\beta_{\mathrm{t}}\right|^{2}\left|\alpha \mathrm{SR}_{\mathrm{t}}\right|^{2} \varepsilon}{\sigma_{\mathrm{v}}^{2}+\left|\alpha_{\mathrm{Rt}} \mathrm{D}\right|^{2}\left|\beta_{2}\right|^{2} \sigma_{\mathrm{wt}}^{2}}\right)$

By substituting Eq. 37 into 28, after a few algebraic manipulations, we can obtain (35) and the proof is complete.

The Monte-Carlo simulation results of the DAF scheme with different numbers of relaying users is shown in Fig. 5. It can be seen that further diversity gains can be achieved by increasing the number of relaying users.

Theorem 4: For a DAF cooperative scheme with $\mathrm{N}(\mathrm{N} \geq 2)$ relaying users, if its transmission rate is $\mathrm{R}$ bits sec $\mathrm{Hz}^{-1}$, its diversity-multiplexing tradeoff is upper bounded by Eq. 38:

$$
\mathrm{d}_{\mathrm{DAF}(\mathrm{N})}(\mathrm{r}) \leq(\mathrm{N}+1)(1-2 \mathrm{r})^{+}
$$

Proof: Similar to the proof of Theorem 2, by analyzing the asymptotic behavior of $\mathrm{I}(\overline{\mathrm{x}}, \overline{\mathrm{y}})$ given by (37), we can conclude that Eq. 39:

$$
\begin{aligned}
& \lim _{\rho \rightarrow \infty} \frac{I(\bar{x}, \bar{y})}{\log \rho} \cong \\
& \frac{1}{2 N} \sum_{t=1}^{N}\left(\max \left\{1-\delta_{S D}, 1-\left(\delta_{\text {SRt }}+\delta S_{t} D\right)\right\}\right)+
\end{aligned}
$$

By defining its outage event set of $\mathrm{O}$ and $\mathrm{O}^{+}$, it is not difficult to calculate that $1-2 \mathrm{r}<\delta_{\mathrm{SD}}<1$ and $\mathrm{N}-2 \mathrm{Nr}<\sum_{\mathrm{t}=1}^{\mathrm{N}}\left(\delta_{\mathrm{SR}}+\delta \mathrm{R}_{\mathrm{t}} \mathrm{D}\right)<\mathrm{N}$. Therefore, the $\mathrm{d}_{\mathrm{DAF}}(\mathrm{N})(\mathrm{r})$ upper bound given by Eq. 38 can be obtained and the proof is complete.

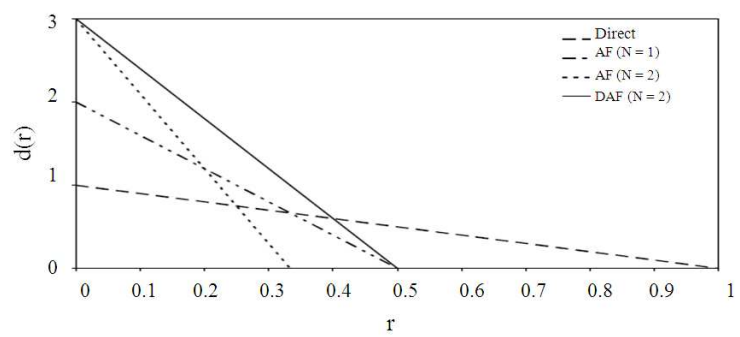

Fig. 4: DMT analysis of the DAF scheme

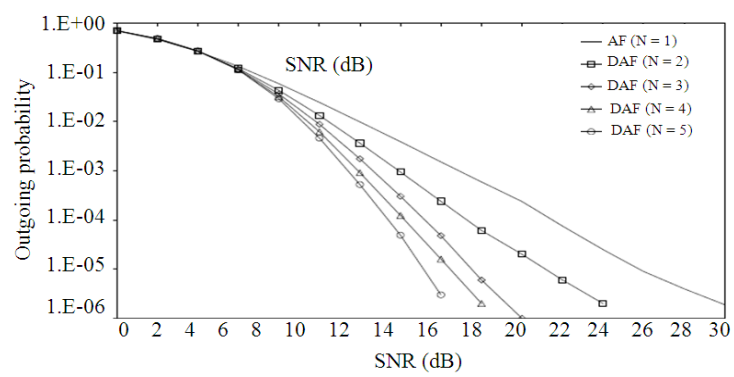

Fig. 5: Outage behavior analysis of DAF scheme with different number of relaying users, $\mathrm{R}=1$ bits sec $\mathrm{Hz}^{-1}$

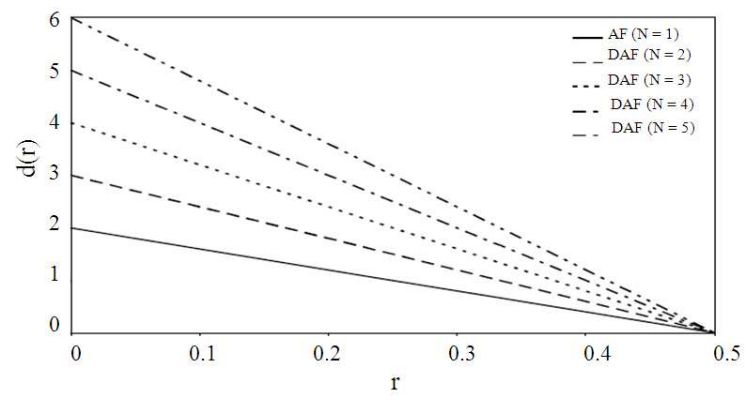

Fig. 6: DMT analysis of DAF scheme with different number of relaying users 
Figure 6 shows the DMT analysis of the DAF scheme with different numbers of relaying users. It varies the achievable diversity gains shown by Fig. 5, in which the outage probability performance is achieved by evaluating the probability model of (35). Note that for the conventional AF scheme with $\mathrm{N}$ relaying users, its DMT performance bound is given by $\mathrm{d}_{\mathrm{AF}}(\mathrm{N})(\mathrm{r}) \leq(\mathrm{N}+1)(1-(\mathrm{N}+1) \mathrm{r})^{+}$. It implies the achievable multiplexing gain is degraded by increasing the number of relaying users. In contrast, the maximal multiplexing gain of the DAF scheme remains unchanged regardless of the number of relaying users and the transmission freedom is maintained.

RSCC coded DAF systems: This system presents a coded cooperative system that integrates the widely used concatenated RSCC codes with the DAF scheme, aiming to show how a practical coded system can exploit the information theoretic gains provided by the DAF scheme.
In the concatenated coding scheme, a RS code and a convolution code are employed as an outer code and an inner code respectively. Correspondingly, in the receiver, the soft-decision Iturbi algorithm (Viterbi, 1967) and the Berlekamp-Massey (BM) algorithm (Massey, 1969) are employed in the decoding process. Integrating with the DAF (N) scheme, the modulated symbols of a codeword are partitioned into $\mathrm{N}$ equal parts for distributed relaying. The coded cooperative system is shown by Fig. 7, where Fig. 7a shows the system structure. It can be noticed that the message partitioning process is assisted by a symbol interleave. Correspondingly, at $\mathrm{D}$, a symbol deinterleaver is employed before the decoding process. The use of interleaving is to enable the Iturbi algorithm to benefit from this diverse transmission (Chen et al., 2010). Figure $7 \mathrm{~b}$ shows the interleaving and deinterleaving processes for a DAF $(\mathrm{N}=2)$ scheme. Now $\mathrm{x}_{\mathrm{k}}(\mathrm{k}=1,2$, ..., $1 / 2$ ) denotes the modulated symbols of a codeword before interleaving.

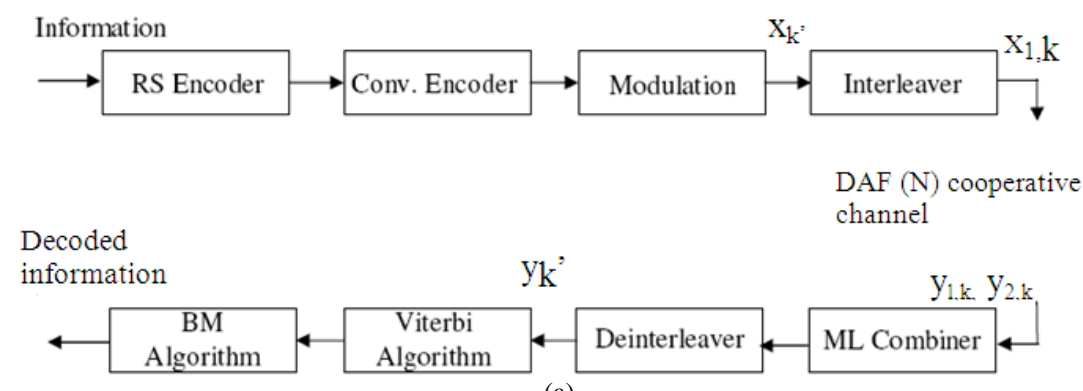

(a)

Interleaving for transmitted symbols

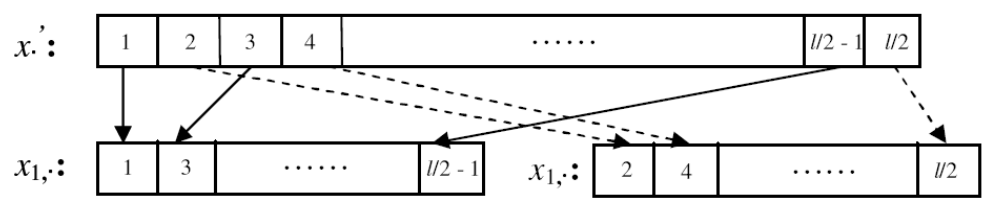

Deinterleaving for received symbols

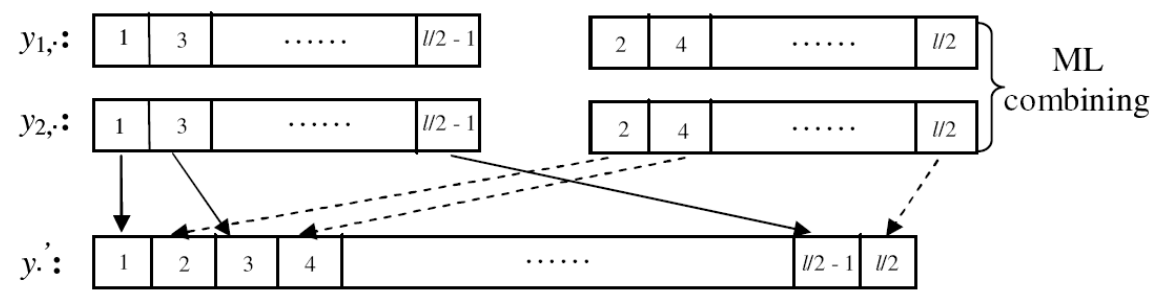

(b)

Fig. 7: RSCC coded DAF system (a): System structure (b): The interleaving and deinterleaving processes 
Am. J. Engg. \& Applied Sci., 4 (4): 607-616, 2011

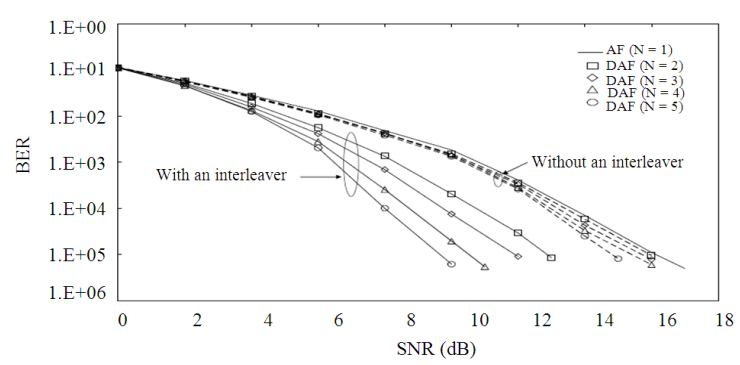

Fig. 8: Performance of $(255,239)$ RS code concatenated with $(171,133)$ convolution code over the DAF channel

It is then interleaved and partitioned into two equal parts ready for transmission. After the DAF cooperative channel, received symbols $\mathrm{y} 1, \mathrm{k}$ and $\mathrm{y} 2, \mathrm{k}$ are combined using the ML combiner (Laneman and Wornell, 2000). The combined symbols are then deinterleaved to form symbols $\mathrm{y}(\mathrm{k}=1,2, \ldots, 1 / 2)$ which will be passed to the Iturbi decoding algorithm.

To ensure a fair comparison of simulation results, the schemes to be compared should provide the same spectral efficiency. Figure 8 shows the performance RSCC coded DAF system. In the system, the inner code is the rate $1 / 2(171,133)$ (in octal form) 64 states convolution code and the outer code is $(255,239)$ RS code. With Quadrature Phase Shift Keying (QPSK) modulation, it achieves a spectral efficiency of 0.47 bits symbol $^{-1}$. The presented simulation results demonstrate that the DAF scheme can enable the coded systems to achieve substantial coding gains. The symbols interleave and deinter leaver plays an important role in maximizing the diversity benefit. For example, in Fig. 8, the DAF $(\mathrm{N}=3)$ scheme with interleave can achieve a $4 \mathrm{~dB}$ performance gain over $\mathrm{AF}(\mathrm{N}=1)$ at a Bit Error Rate (BER) of $10^{-5}$. Without interleave, the performance gain is only $0.5 \mathrm{~dB}$. However, it is important to mention that symbol interleaving may not be necessary for codes which do not utilize trellis decoding. The simulation results demonstrate that the proposed cooperative scheme can indeed benefit a practical coded system and therefore is suitable to be considered for practical applications.

\section{CONCLUSION}

A novel distributed amplify-and-forward cooperation scheme was proposed. For each user in the DAF cooperative uses half of their transmission for transmitting information, while the other half for relaying information. Therefore, it is able to maintain the same transmission freedom allowed in the conventional AF cooperative network. Outage behavior of the DAF scheme was analyzed, showing $5 \mathrm{~dB}$ diversity gain can be achieved over the AF scheme at outage probability of $10^{-5}$. Further diversity gains can be achieved by increasing the number of users. A DMT analysis of the DAF scheme was not only varied that the DAF scheme can achieve a higher diversity gain than the AF scheme, but also justice it can maintain the same transmission freedom by showing its ability to maintain a constant multiplexing gain. However, it is also worthwhile to point out that the DAF scheme requires higher system complexity, since better synchronization and more complex channel information shall be provided among cooperative users. A RSCC coded system was proposed in order to validate the performance advantage provided by the DAF scheme. It was shown that with the assistance of the symbol interleave, significant performance gain can be yielded over the existing AF scheme. Therefore, the proposed DAF scheme is a simple and well-performing distributed cooperation strategy that is suitable for practical

\section{ACKNOWLEDGMENT}

This project is sponsored by the Engineering and Physical Science Research Council (EPSRC) under Grant

\section{REFERENCES}

Alamouti, S.M., 1998. A simple transmit diversity technique for wireless communications. IEEE J. Selected Areas Commun., 16: 1451-1458. DOI: 10.1109/49.730453

Azarian, K., H.E. Gamal and P. Schniter, 2005. On the achievable diversity-multiplexing tradeoff in halfduplex cooperative channels. IEEE Trans. Inform. Theory, 51: 4152-4172. DOI: 10.1109/TIT.2005.858920

Chatzigeorgiou, I., W. Guo, I J. Wassell and R.A. Carrasco, 2008. Comparison of cooperative schemes using joint channel coding and high-order modulation. Proceeding of the 3rd International Symposium on Communications, Control and Signal Processing, Mar. 12-14, IEEE Xplore Press, St Julians, pp: 994-998. DOI: 10.1109/ISCCSP.2008.4537368

Chen, L., R. Carrasco and I. Wassell, 2010. Distributed amplify-and-forward with ring-tcm codes. Proceeding of the 7th IEEE Consumer Communications and Networking Conference (CCNC), Jan. 9-12, IEEE Xplore Press, Las Vegas, NV., pp: 1-5. DOI: 10.1109/CCNC.2010.5421691

Chen, L., R.A. Carrasco and I. Wassell, 2011. Distributed amplify-and-forward cooperation through message partitioning. IEEE Trans. Veh. Technol., 60: 3054-3065. DOI: 10.1109/TVT.2011.2162756 
Chen, L., R.A. Carrasco, S. LeGoff and I.J. Wassell, 2009. Cooperative amplify-and-forward with trellis coded modulation. Proceeding of the IEEE Wireless Communications and Networking Conference, Apr. 5-8, IEEE Xplore Press, Budapest, pp: 1-5. DOI: 10.1109/WCNC.2009.4917641

Chen, L., R.A. Carrasco, V. Ganepola, S. LeGoff and I.J. Wassell, 2008. Comparative investigation of coded cooperative communication systems. Proceeding of the 6th International Symposium on Communication Systems, Networks and Digital Signal Processing, Jul. 25-25, IEEE Xplore Press, Graz, pp: 58-62. DOI: 10.1109/CSNDSP.2008.4610729

Hunter, T.E. and A. Nosratinia, 2006. Diversity through coded cooperation. IEEE Trans. Wireless Commun., 5 : 283-289. DOI: 10.1109/TWC.2006.1611050

Laneman, J.N. and G.W. Wornell, 2000. Energyefficient antenna sharing and relaying for wireless networks. Proceeding of the IEEE Wireless Communications and Networking Confernce, Sep. 23-28, IEEE Xplore Press, Chicago, IL., pp: 7-12. DOI: 10.1109/WCNC.2000.904590

Laneman, J.N. and G.W. Wornell, 2003. Distributed space-time-coded protocols for exploiting cooperative diversity in wireless networks. IEEE Trans. Inform. Theory, 49: 2415-2425. DOI: 10.1109/TIT.2003.817829
Laneman, J.N., D.N.C. Tse and G.W. Wornell, 2004. Cooperative diversity in wireless networks: Efficient protocols and outage behavior. IEEE Trans. Inform. Theory, 50: 3062-3080. DOI: 10.1109/TIT.2004.838089

Massey, J., 1969. Shift-register synthesis and BCH decoding. IEEE Trans. Inform. Theory, 15: 122127. DOI: 10.1109/TIT.1969.1054260

Stefanov, A. and E. Erkip, 2004. Cooperative coding for wireless networks. IEEE Trans. Commun., 52: 1470-1476. DOI: 10.1109/TCOMM.2004.833070

Ungerboeck, G., 1982. Channel coding with multilevel/phase signals. IEEE Trans. Inform. Theory, 28: 55-67. DOI: 10.1109/TIT.1982.1056454

Viterbi, A., 1967. Error bounds for convolutional codes and an asymptotically optimum decoding algorithm. IEEE Trans. Inform. Theory, 13: 260269. DOI: $10.1109 /$ TIT.1967.1054010

Zheng, L. and D.N.C. Tse, 2003. Diversity and multiplexing: A fundamental tradeoff in multipleantenna channels. IEEE Trans. Inform. Theory, 49: 1073-1096. DOI: 10.1109/TIT.2003.810646 